\title{
Fluidisation de particules de bois en lit mixte : faisabilité et contrôle du procédé
}

\author{
Jean-Louis Tangier ${ }^{\mathrm{a}}$, Riad Benelmir et Mahamed El Ganaoui \\ Laboratoire LERMAB, Faculté des Sciences, 54506 Vandœuvre lès Nancy, France
}

Reçu le 28 décembre 2011, accepté le 6 janvier 2012

\begin{abstract}
Résumé - Le séchage cinétique des particules en bois dans un lit de sable fluidisé est étudié. Le réacteur est constitué d'une colonne de verre cylindrique où la fluidisation des particules de sable est obtenue grâce à un débit d'air sec, filtré, dont la pression est ajustée par un détendeur et dont le débit est mesuré. Le sable a un diamètre moyen de $0,155 \mathrm{~mm}$ et une masse volumique de $2650 \mathrm{~kg} . \mathrm{m}^{-3}$. La température et l'humidité relative de l'air sont respectivement mesurées par des thermocouples et par un hygromètre capacitif à film mince mesurant l'humidité absolue de l'air à l'admission et à la sortie du lit fluidisé. L'installation expérimentale est conçue pour évaluer la valeur instantanée de l'humidité du bois. Le taux de séchage augmente avec la température et la masse des particules de bois. Ces comportements sont examinés en tenant compte des différents paramètres (température, vitesse de l'air, humidité initiale du bois).
\end{abstract}

Mots clés : Lit fluidisé mixte / séchage de particules de bois / paramètres de fluidisation / quantité de bois fluidisable

\begin{abstract}
Fluidization of wood particles in a mixed bed: faisability and process control. We study the drying kinetics of wood particles in a sand fluidized bed. The investigation domain is a cylindrical glass column where fluidisation of sand particles is obtained by means of a dry air flow. This air is cleaned through two filters. The bed's sand has an average diameter of $0.155 \mathrm{~mm}$ and a density of $2650 \mathrm{~kg} \cdot \mathrm{m}^{-3}$. The temperature and the relative humidity of the air flow are respectively measured by chromel-alumel thermocouples and by a capacitive hygrometer allowing the calculation of air absolute humidity at the inlet and outlet of the fluidized bed. The experimental setup is designed in order to evaluate the wood instantaneous absolute humidity. The drying rate increases with the temperature, and the mass of wood particles temperature. These behaviors have been analyzed according to various parameters (inlet air temperature and velocity, initial wood moisture content).
\end{abstract}

Key words: Fluidised bed joint / drying of wood particles / settings fluidization / amount of wood fluidizable

\section{Introduction}

La valorisation du bois sous forme de plaquettes, de copeaux ou de fines particules implique des phases de traitement thermique telles que le séchage, la torréfaction, la carbonisation ou la gazéification. Lors de telles opérations, l'apport d'énergie thermique se fait généralement par échange entre une phase gazeuse chauffée et le matériau à traiter avec des coefficients de transfert faibles.

Les transferts de chaleur et de masse sont très intenses dans un lit fluidisé compte tenu de la turbulence engendrée par les particules en mouvement. Cette technologie est particulièrement efficace pour sécher, à

\footnotetext{
a Auteur pour correspondance :

tanguier@lermab.uhp-nancy.fr
}

température modérée, des produits présentant une humidité importante (silice [1], céramique [2], résines [3], blé [4], etc.) mais aussi des réactions chimiques [5-7].

Dans le cas de particules, dont les caractéristiques (taille, masse volumique, sphéricité ...) ne permettent pas la fluidisation directe, il a été montré [9-11] que la fluidisation peut être obtenue indirectement par l'ajout de corps solides inertes, possédant une bonne aptitude à la fluidisation.

Cette technique a été testée avec des particules de bois pour vérifier la faisabilité de l'opération. L'objectif visé est de les soumettre à différents types de traitements thermiques (séchage, torréfaction, carbonisation) à des niveaux de température différents.

Le banc expérimental ayant été conçu et réalisé, le lit fluidisé est caractérisé sur le plan hydrodynamique, puis 


\section{Nomenclature}

\begin{tabular}{|lll|}
\hline$d_{\mathrm{p}}$ & diamètre moyen des particules de bois & $\mathrm{m}$ \\
$h$ & hauteur de colonne d'eau & $\mathrm{m}$ \\
$g$ & accélération de la pesanteur & $\mathrm{m}_{\mathrm{s}}{ }^{-2}$ \\
$H_{\mathrm{bois}}$ & humidité du bois & $\%$ \\
$H_{\mathrm{mf}}$ & hauteur du lit fluidisé au minimum de fluidisation & $\mathrm{m}$ \\
$H_{\mathrm{mx}}$ & hauteur maximale du lit fluidisé & $\mathrm{m}$ \\
$U_{\mathrm{mf}}$ & vitesse minimale de fluidisation & $10^{-2} \cdot \mathrm{m} \cdot \mathrm{s}^{-1}$ \\
$V$ & vitesse de l'air & $\mathrm{m} \cdot \mathrm{s}^{-1}$ \\
$\varepsilon$ & porosité du lit fluidisé & \\
$\mu$ & viscosité dynamique du fluide & $\mathrm{kg} \cdot \mathrm{m}^{-1} \cdot \mathrm{s}^{-1}$ \\
$\rho_{\mathrm{f}}$ & masse volumique du fluide & $\mathrm{kg} \cdot \mathrm{m}^{-3}$ \\
$\rho_{\mathrm{p}}$ & masse volumique des particules de bois & $\mathrm{kg} \cdot \mathrm{m}^{-3}$ \\
\hline
\end{tabular}

avec particules de bois. L'analyse des résultats permet alors d'établir les caractéristiques et les limites de fonctionnement d'un lit mixte sable/bois.

\section{Dispositif expérimental}

La conception, puis la réalisation du dispositif expérimental représentent une phase importante pour fluidiser des particules de bois dans un lit de particules inertes. La répartition est vérifiée à l'aide d'un tube en verre (corps du réacteur). Cette option a imposé les choix techniques du dispositif expérimental (Fig. 1) qui se compose de trois constituants principaux entre l'alimentation en air en amont et l'instrumentation en aval.

Le réacteur se compose de la colonne en verre, du diffuseur et d'une cuve en acier inoxydable. L'alimentation en air est assurée par un compresseur autonome qui délivre le fluide sous une pression de 7 bars. La mesure du débit et de l'humidité de l'air se fait au niveau du conduit d'évacuation de $19 \mathrm{~mm}$ grâce à un diaphragme mince (9,6 mm d'orifice). Différentes sondes de température sont également installées dans le lit.

1. La colonne en verre, qui constitue le volume contenant les particules à fluidiser, a une hauteur de $850 \mathrm{~mm}$ pour un diamètre intérieur de $230 \mathrm{~mm}$. Elle est surmontée par une bride qui comporte l'évacuation de l'air et les orifices nécessaires à l'introduction des différents capteurs. L'assemblage de ces différents éléments est assuré par des tirants.

La base du système est composée d'une cuve de $400 \mathrm{~mm}$ de hauteur et de $230 \mathrm{~mm}$ de diamètre intérieur. Cet élément peut être équipé de résistances électriques et a pour rôle d'homogénéiser en température le flux d'air entrant dans la colonne.

Il est surmonté du diffuseur qui a pour fonction de maintenir les particules dans la colonne tout en assurant une diffusion homogène de l'air. Il est composé de deux tamis de $100 \mu \mathrm{m}$, séparés par une feuille poreuse (en papier). Pour garantir sa tenue mécanique, cet ensemble est monté entre deux grilles métalliques dont le maillage est de $50 \mu \mathrm{m}$. Cet ensemble est inséré dans un logement ménagé entre la cuve et la bride d'assemblage de la colonne en verre.
2. Un détendeur permet d'ajuster à 0,7 bar la pression d'admission d'air qui a été préalablement séché et déshuilé par passage sur des filtres. Le débit d'air est alors contrôlé par un débitmètre à flotteur avant d'être introduit dans le réchauffeur qui a été dimensionné pour assurer un débit de $10 \mathrm{~m}^{3} \cdot \mathrm{h}^{-1}$ d'air à $160{ }^{\circ} \mathrm{C}$. L'air est ensuite introduit à la base de la cuve composant la base du système qui sert également de volume tampon.

La différence de pression générée par le diaphragme est lue en continu sur un manomètre en $U$. La conversion des millimètres de colonne d'eau en vitesse d'air dans le dispositif est obtenue à l'aide de la relation d'étalonnage établie à partir des mesures réalisées avec un anémomètre à moulinet et conduit à une corrélation du type $V=0,203 \mathrm{~h}^{1 / 2}\left(\mathrm{~m} . \mathrm{s}^{-1}\right)$.

Une sonde mobile de mesure de pression, reliée à un manomètre en $U$, permet de mesurer la variation de pression statique dans le lit fluidisé. La différence de pression donne la perte de charge. L'extrémité de cette canne est pourvue d'un filtre de maillage inférieur au diamètre des grains de sable pour éviter qu'ils ne remplissent la canne faussant les mesures.

3. Une sonde de mesure de température équipée de cinq thermocouples de type $K$, chemisés et de $1 \mathrm{~mm}$ de diamètre permet de mesurer l'évolution de la température radiale dans le lit fluidisé en partant de l'axe vers l'extérieur par pas de 2,5 cm. Cette sonde, également mobile, autorise la mesure des températures radiales à différentes profondeurs d'immersion dans le sable. Elle permettra ultérieurement de vérifier l'homogénéité en température du lit.

Une sonde hygrométrique (capteur capacitif à film mince) est également prévue en aval du diaphragme. Elle permet de suivre l'évolution de l'hygrométrie relative de l'air extrait du réacteur en fonction du temps.

\section{Hydrodynamique du système sans particules}

Ce paragraphe est consacré à la détermination des principaux paramètres de fluidisation (minimum de fluidisation et taux d'expansion) avec le sable seul. 

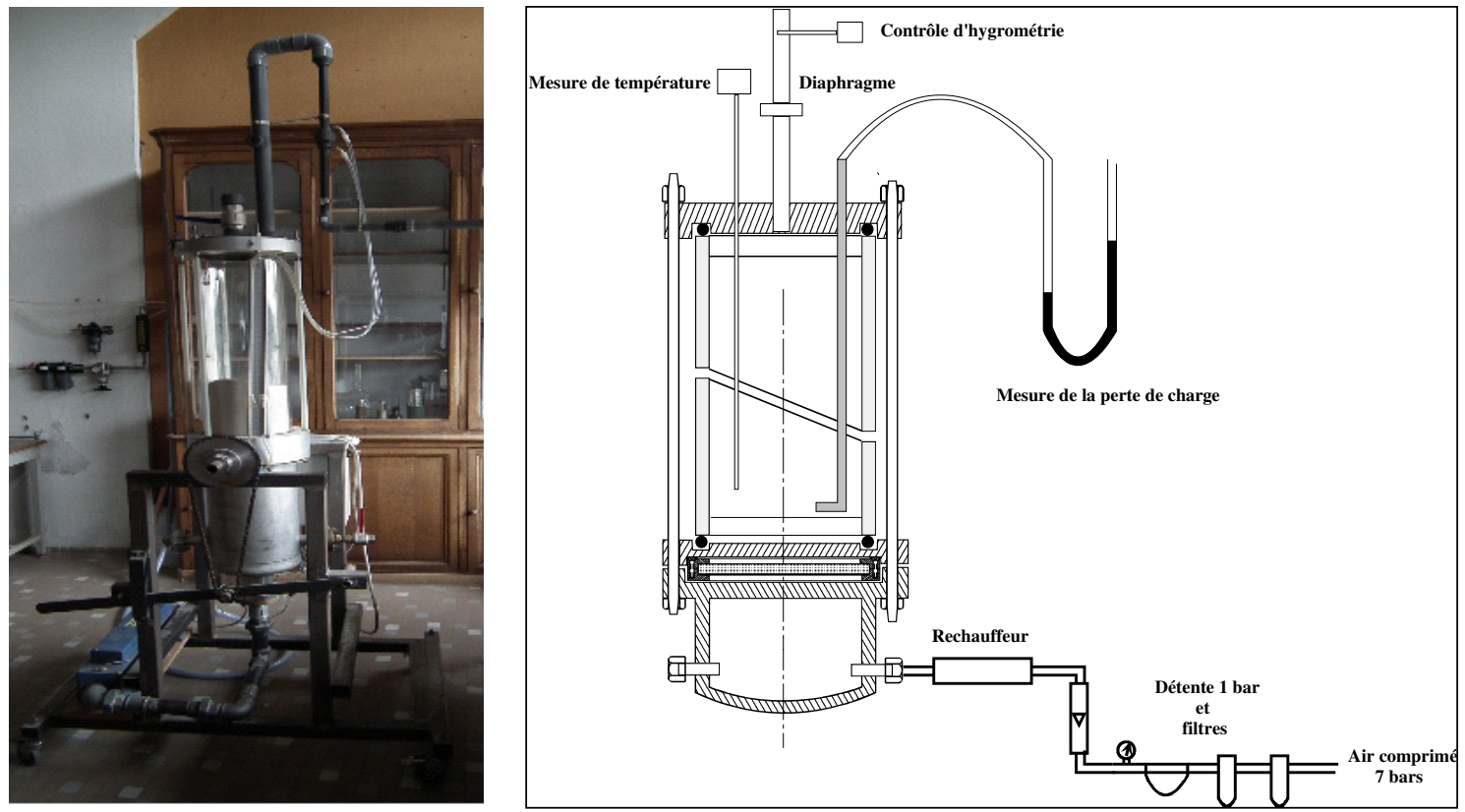

Fig. 1. Vue et schéma de l'installation.

\subsection{Caractérisation du sable}

Plus le matériau à fluidiser est dense, plus la force nécessaire pour le mettre en mouvement est importante. La masse volumique du sable est donc un paramètre important. En utilisant un pycnomètre à eau, la masse volumique du sable est évaluée à $2650 \mathrm{~kg} . \mathrm{m}^{-3}$.

La dimension des particules de sable est également un paramètre important dans le cadre de la fluidisation, en particulier lorsque les particules sont non sphériques. La répartition granulométrique est donnée dans le tableau 1. Un diamètre médian en masse est retenu comme paramètre de travail pour caractériser les particules de sable. Il est égal à l'ouverture du tamis correspondant à $50 \%$ du pourcentage pondéral cumulé soit $d_{m}=162 \mu \mathrm{m}$.

\subsection{Perte de charge et vitesse minimale de fluidisation}

L'évolution de la perte de charge en fonction de la vitesse de l'air de fluidisation pour différentes quantités de sable dans le réacteur est représentée sur la figure 2. Elle permet également d'identifier la vitesse minimale de fluidisation.

Ces mesures permettent de vérifier que la perte de charge est proportionnelle à la hauteur du lit de sable. La perte de charge (ramenée à l'unité de hauteur du lit), est quasi constante à partir du minimum de fluidisation.

Dans nos conditions opératoires, l'évolution de la perte de charge dans le lit fixe et le lit fluidisé est résumée respectivement par $\Delta P / H=5344 . U$ pour un lit fixe et par $\Delta P / H=13077+97 . U$ pour un lit fluidisé.

L'abscisse de l'intersection de ces deux droites donne la vitesse minimale de fluidisation soit : $U_{\mathrm{mf}}=$ $2,49 \mathrm{~cm} . \mathrm{s}^{-1}$.
En assimilant les grains de sable à des particules sphériques, nous pouvons utiliser la relation de la référence [12] qui relie la vitesse minimale de fluidisation au nombre de Galilée $G a=d_{\mathrm{p}}^{3} \rho_{\mathrm{f}} g\left(\rho_{\mathrm{s}}-\rho_{\mathrm{f}}\right) / \mu$, soit :

$$
\begin{aligned}
R e_{\mathrm{mf}} & =a G a^{b} \quad \text { ou encore : } \\
U_{\mathrm{mf}} & =a \frac{\mu}{\rho_{\mathrm{g}} d_{\mathrm{p}}} G a^{b}
\end{aligned}
$$

où $a$ et $b$ sont des constantes de régression.

Par ailleurs, Wen et $\mathrm{Yu}[9]$ ont montré que :

$$
U_{\mathrm{mf}}=\frac{\mu}{\rho_{\mathrm{g}} d_{\mathrm{p}}}\left[\frac{(33,7)^{2}+0,0408 d_{\mathrm{p}}^{3} \rho_{\mathrm{g}}\left(\rho_{\mathrm{p}}-\rho_{\mathrm{g}}\right) g}{\mu^{2}}\right]^{0,5}
$$

Dans le cas des faibles nombres de Reynolds, $\left(R e_{\mathrm{p}, \mathrm{mf}}<\right.$ 20) (ce qui est notre cas $R e_{\mathrm{p}, \mathrm{mf}}=0,255$ ), on a :

$$
U_{\mathrm{mf}}=\frac{d_{\mathrm{p}}^{2}\left(\rho_{\mathrm{p}}-\rho_{\mathrm{g}}\right) g}{1650 \mu}
$$

Une expression similaire à l'équation (1) pour laquelle le coefficient $b=1$ est obtenue. En partant des résultats expérimentaux nous pouvons alors définir la constante $\ll a »$ qui est obtenue par optimisation en minimisant une fonction erreur relative entre la valeur calculée et la valeur mesurée. Dans ces conditions :

$$
U_{\mathrm{mf}}=\frac{d_{\mathrm{p}}^{2}\left(\rho_{\mathrm{p}}-\rho_{\mathrm{g}}\right) g}{1441 \mu}
$$

Les expressions proposées par différents auteurs font toutes apparaître le diamètre $\left(d_{\mathrm{p}}\right)$ des particules. Avec ces corrélations, une dispersion de l'ordre de $7 \%$ est obtenue (Tab. 2). 
J.L. Tanguier et al. : Mechanics \& Industry 13, 69-76 (2012)

Tableau 1. Répartition granulométrique en masse du sable.

\begin{tabular}{cccccccc}
\hline Classe $(\mu \mathrm{m})$ & $>425$ & $300-425$ & $212-300$ & $150-212$ & $106-150$ & $75-106$ & $<75$ \\
\hline Fraction massique $(\%)$ & 0 & 0,3 & 7,9 & 52,6 & 35,9 & 2,9 & 0,4 \\
\hline
\end{tabular}

Tableau 2. Vitesse minimale théorique de fluidisation.

\begin{tabular}{|c|c|c|c|}
\hline Auteurs & Corrélations & $V_{\mathrm{mf}}\left(\mathrm{m} \cdot \mathrm{s}^{-1}\right)$ & Écart relatif $(\%)$ \\
\hline Présente étude & $U_{\mathrm{mf}}=\frac{d_{\mathrm{p}}^{2}\left(\rho_{\mathrm{p}}-\rho_{\mathrm{g}}\right) g}{1441 \mu}$ & $2,49 \times 10^{-2}$ & - \\
\hline Goroshko et al. [10] & $U_{\mathrm{mf}}=\frac{\mu}{d \rho_{\mathrm{f}}}\left(\frac{G a}{1400+5.2 \sqrt{G a}}\right)$ & $2,46 \times 10^{-2}$ & $-1,6$ \\
\hline Pillai et al. [11] & $U_{\mathrm{mf}}=\frac{7,01 \times 10^{-4} d^{2}\left(\rho-\rho_{\mathrm{f}}\right) g}{\mu}$ & $2,59 \times 10^{-2}$ & 3,6 \\
\hline Baeyens et al. [12] & $U_{\mathrm{mf}}=\frac{9,125 \times 10^{-4}\left(\rho-\rho_{\mathrm{f}}\right)^{0,934} g^{0,934} d^{1,8}}{\mu^{0,87} \rho_{\mathrm{f}}^{0,066}}$ & $2,36 \times 10^{-2}$ & $-5,6$ \\
\hline Richardson et al. [13] & $U_{\mathrm{mf}}=\frac{\mu}{d \rho_{\mathrm{f}}}\left[\left(25,7^{2}+0,0365 G a\right)^{0,5}-25,7\right]$ & $2,64 \times 10^{-2}$ & 5,6 \\
\hline Thonglimp et al. [8] & $U_{\mathrm{mf}}=\frac{\mu}{d \cdot \rho_{\mathrm{f}}} \cdot\left[7,169 \times 10^{-4} \cdot G a^{0,98}\right]$ & $2,35 \times 10^{-2}$ & 6,0 \\
\hline Bena et al. [14] & $U_{\mathrm{mf}}=\frac{\mu}{d \rho_{\mathrm{f}}} \frac{1,38 \times 10^{-3} G a}{(G a+19)^{0,11}}$ & $2,63 \times 10^{-2}$ & 5,2 \\
\hline Leva et al. [15] & $U_{\mathrm{mf}}=\frac{7,169 \times 10^{-4} d^{1,82}\left(\rho-\rho_{\mathrm{f}}\right)^{0,94} g}{\rho^{0,06} \mu^{0,88}}$ & $2,10 \times 10^{-2}$ & 16 \\
\hline
\end{tabular}

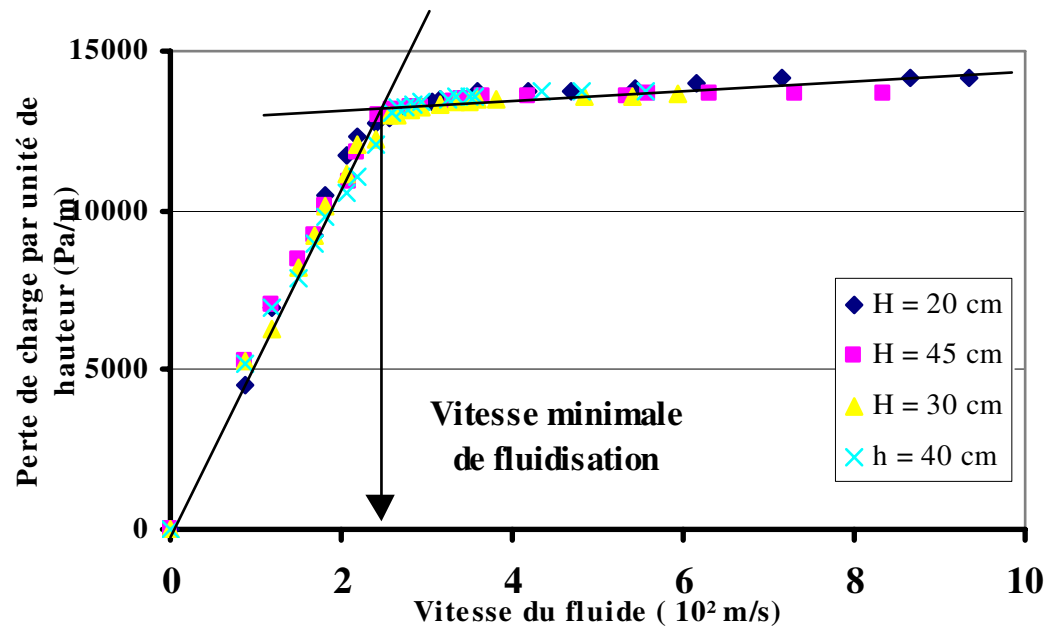

Fig. 2. Perte de charge et vitesse minimale de fluidisation.

\subsection{Porosité et taux d'expansion}

Les résultats expérimentaux donnent une valeur de la porosité au minimum de fluidisation telle que $\varepsilon_{\mathrm{mf}}=0,47$. Au-delà, la porosité augmente linéairement avec le débit d'air :

$$
\varepsilon=\varepsilon_{\mathrm{mf}}+0,01143\left(U-U_{\mathrm{mf}}\right)
$$

L'expansion du lit fluidisé varie linéairement avec la vitesse et est indépendante de la hauteur initiale de sable :

$$
H_{\mathrm{mx}} / H_{\mathrm{mf}}=1-\frac{0,11}{\sqrt{g d_{\mathrm{p}}}}\left(U-U_{\mathrm{mf}}\right)
$$

Les résultats sont en accord avec ceux de la littérature avec des écarts inférieurs à $10 \%$ (Tab. 3).

\section{Hydrodynamique du système avec particules de bois}

Trois lots de particules de bois sont sélectionnés (Tab. 4). En dépit de leurs formes irrégulières, nous assimilerons ces particules à des parallélépipèdes. La fluidisation est la possibilité de suspendre les particules 
Tableau 3. Taux d'expansion théorique et expérimental en fonction de la vitesse.

\begin{tabular}{|c|c|c|c|c|c|c|}
\hline & & \multicolumn{5}{|c|}{$U-U_{\mathrm{mf}}\left({\left.\mathrm{M} . \mathrm{s}^{-1}\right)}^{-1}\right.$} \\
\hline & & 0 & 2,5 & 5 & 7,5 & 10 \\
\hline Hsiung et & $\varepsilon=\varepsilon_{\mathrm{mf}}\left({\frac{R e-K}{R e_{\mathrm{mf}}-K}}^{0,28}\right)$ & 1 & 1,108 & 1,213 & 1,318 & 1,425 \\
\hline Thodos [16] & avec $K=0,216 R e_{\mathrm{mf}}-0,35$ & & $(2,5 \%)$ & $(4,5 \%)$ & $(8 \%)$ & $(10 \%)$ \\
\hline $\begin{array}{l}\text { Lewis et } \\
\text { Bowermann }[17]\end{array}$ & $\frac{H_{\mathrm{mx}}}{H_{\mathrm{mf}}}=1+\frac{0,0106 U-U_{\mathrm{mf}}}{\sqrt{g \cdot d_{\mathrm{p}}}}$ & 1 & $\begin{array}{l}1,066 \\
(1,2 \%)\end{array}$ & $\begin{array}{c}1,133 \\
(2,3 \%)\end{array}$ & $\begin{array}{c}1,199 \\
(1,7 \%)\end{array}$ & $\begin{array}{c}1,266 \\
(1,8 \%)\end{array}$ \\
\hline $\begin{array}{l}\text { Babu et al. } \\
\qquad[18]\end{array}$ & $\frac{H_{\mathrm{mx}}}{H_{\mathrm{mf}}}=1+0,450 \frac{d_{\mathrm{p}}}{D_{\mathrm{lit}}}\left[\frac{\left(U-U_{\mathrm{mf}}\right)}{U_{\mathrm{mf}}}\right]^{0,738}\left(\frac{\rho_{\mathrm{p}}}{\rho_{\mathrm{g}}}\right)^{0,376}$ & 1 & $\begin{array}{c}1,005 \\
(6,9 \%)\end{array}$ & $\begin{array}{c}1,009 \\
(13 \%)\end{array}$ & $\begin{array}{c}1,012 \\
(17 \%)\end{array}$ & $\begin{array}{l}1,0153 \\
(21 \%)\end{array}$ \\
\hline Expérimental & $\frac{H_{\mathrm{mx}}}{H_{\mathrm{mf}}}=1-\frac{0,00114}{\sqrt{g d_{\mathrm{p}}}}\left(U-U_{\mathrm{mf}}\right)$ & 1 & 1,08 & 1,16 & 1,22 & 1,29 \\
\hline
\end{tabular}

Tableau 4. Caractéristiques des particules de bois.

\begin{tabular}{cccc}
\hline & Lot 1 & Lot 2 & Lot 3 \\
\hline Essence & hêtre & hêtre & peuplier \\
\hline Forme & parallélépipède & sciure fine & bâtonnet \\
\hline Dimension & cubes de $4 \mathrm{~mm}$ & grains de $2,5 \mathrm{~mm}$ & épaisseur $2 \mathrm{~mm}$ largeur $3 \times$ épaisseur \\
& de côté & de diamètre & longueur $5 \times$ largeur \\
\hline$\rho\left(\mathrm{kg} \cdot \mathrm{m}^{-3}\right)$ & 600 & 600 & 400 \\
\hline
\end{tabular}

dans une couche de sable fluidisé; ce qui sera vérifié expérimentalement.

\subsection{Quantité de bois fluidisable}

Différents essais montrent que les particules de bois se mélangent difficilement au sable du lit fluidisé. Ceci est probablement dû à la différence de masse volumique entre le bois et le sable. On observe qu'il existe une quantité maximale de bois susceptible de se mélanger de façon homogène.

Pour la déterminer, des quantités de bois croissantes sont introduites pour diverses hauteurs de sable et nous avons défini que le lit mixte n'était plus homogène lorsque l'épaisseur de bois qui demeure en surface du lit fluidisé atteint $1 \mathrm{~cm}$.

La figure 3, montre que la quantité maximale, en volume, de particules de hêtre (lots 1 et 2) qui peut être fluidisé de façon homogène correspond à 0,55 fois la quantité de sable initiale $(0,45$ pour le peuplier (lot 3$))$.

En termes de masse, cela correspond à $25 \%$ pour le hêtre et $14 \%$ pour le peuplier.

\subsection{Perte de charge et vitesse minimale de fluidisation}

La figure 4 donne l'évolution de la perte de charge mesurée pour le lot No. 1. On constate que l'adjonction de bois a pour effet d'augmenter, à vitesse d'air égale, la perte de charge de 8 à $18 \%$ dans le lit lorsque la hauteur de bois passe de 8 à $20 \mathrm{~cm}$. La vitesse minimale de fluidisation diminue avec la hauteur de bois (avec $8 \mathrm{~cm}$ de bois, elle est égale à $2,32 \times 10^{-2} \mathrm{~m} . \mathrm{s}^{-1}$ pour atteindre $1,95 \times 10^{-2} \mathrm{~m} \cdot \mathrm{s}^{-1}$ avec $20 \mathrm{~cm}$ de bois).

Les résultats obtenus avec le lot No. 2 sont tout à fait comparables à ceux obtenus avec le lot No. 1; les morceaux de bois peuvent être assimilés à des cubes. Les résultats expérimentaux sont en accord avec ceux de Strijbos [19] qui a montré que la vitesse de fluidisation du mélange (sable et bois) est inférieure à celle du sable seul. Ce phénomène peut s'expliquer par le fait que la masse volumique globale du mélange diminue alors que le diamètre équivalent du mélange augmente.

\subsection{Séchage des particules}

Pour suivre en continu l'évolution de l'humidité, le flux d'air sortant du réacteur est canalisé afin de mesurer l'humidité relative de l'air. En mesurant également le débit et la température de ce flux d'air, il est alors possible d'évaluer la masse d'eau extraite du système.

Pour vérifier le bien fondé de cette méthode, nous avons procédé à divers essais en introduisant une quantité d'eau connue dans le réacteur préalablement mis à l'équilibre (température et humidité).

Dans un premier temps, une masse connue d'eau (200 g et 400 g) est injectée. La masse en eau déduite des mesures d'humidité relative de l'air en sortie du réacteur conduit à des écarts maximum de 5 à $6 \%$ de la quantité initiale.

Dans un second temps, nous avons humidifié le bois du lot 1 avec une quantité connue d'eau (adsorption) soit 


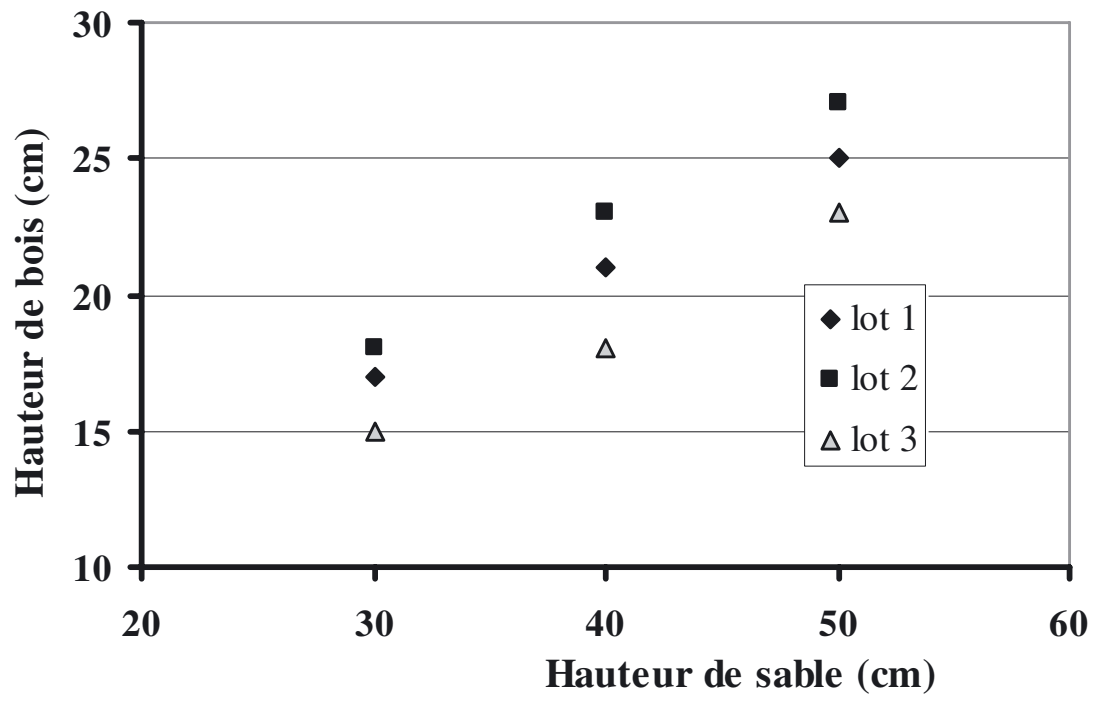

Fig. 3. Hauteur maximale de bois pouvant être fluidisée.
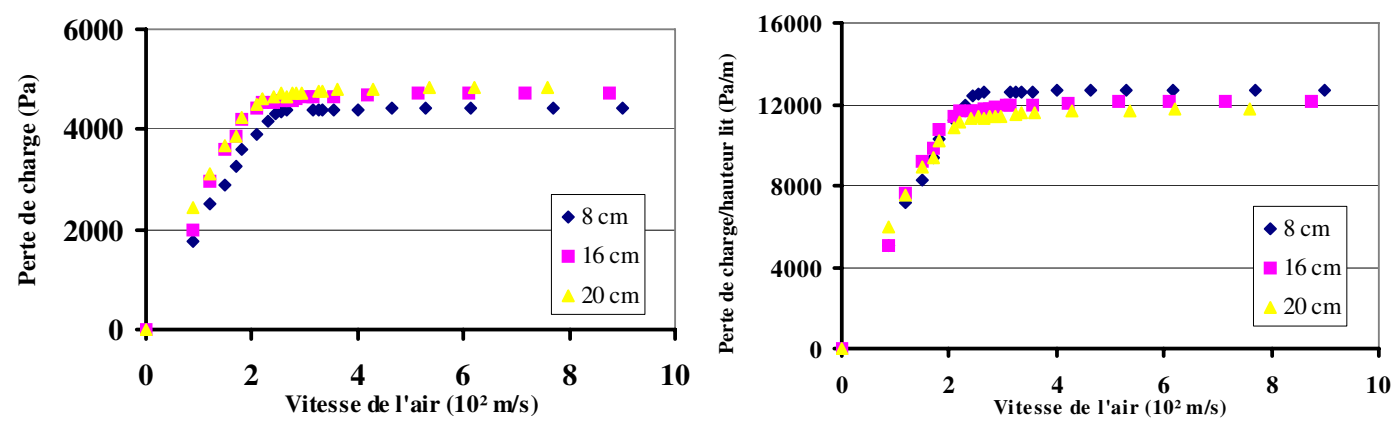

Fig. 4. Perte de charge de cubes de hêtre pour le lot 1 (gauche) et lot 2 (droite).

\section{Humidité relative et Eau cumulée}

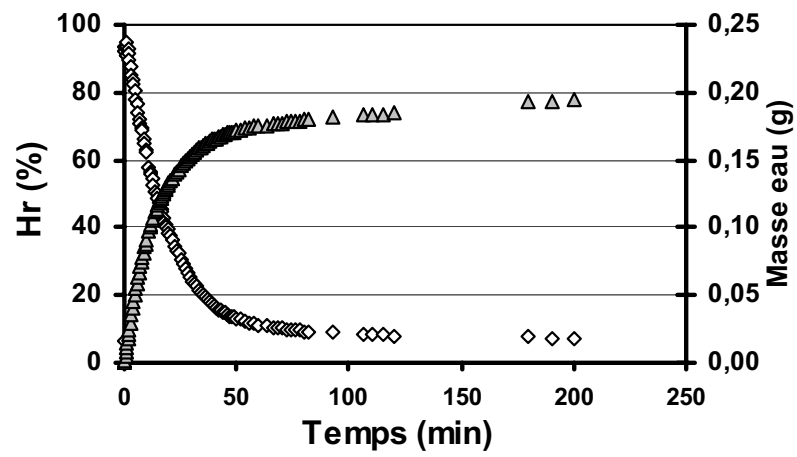

Fig. 5. Évolution de l'humidité relative de l'air extrait du lit en fonction du temps.

$300 \mathrm{~g}$ de bois sec et $200 \mathrm{~g}$ d'eau. Les résultats obtenus conduisent à un écart en masse d'eau recueillie de $\pm 2,5 \%$ avec une évolution des paramètres donnée par la figure 5 .

Cette technique semble donc intéressante pour évaluer globalement la charge en eau évacuée. Par contre elle ne permet pas d'évaluer l'état de séchage du bois dans la charge de sable sauf si on admet que le séchage du bois suit une évolution similaire.

Les essais ont été réalisés uniquement avec les lots 1 et 2. En ce qui concerne le lot 3, nous avons montré qu'il n'y avait pas de mélange intime entre le bois et le sable. Lors de différents essais nous avons fait varier les paramètres expérimentaux tels que l'humidité initiale du bois, la masse de bois introduite, la température du sable et de l'air ainsi que le débit d'air.

L'analyse des résultats permet tout d'abord de montrer que la taille des particules de bois, avec des conditions opératoires identiques, n'a que très peu d'influence quant au séchage du bois. On considère donc que le comportement du lot 1 est identique à celui du lot 2 .

\section{Conclusion}

La faisabilité de la fluidisation de particules de bois a été établie, ce qui est un résultat important au vu de l'étendue des applications possibles (optimisation de la combustion par exemple). Elle est d'autant plus facile que les particules sont de forme cubique ou sphérique.

Avec le hêtre (lots 1 et 2), les quantités de bois qui peuvent être fluidisées correspondent à un volume proche 

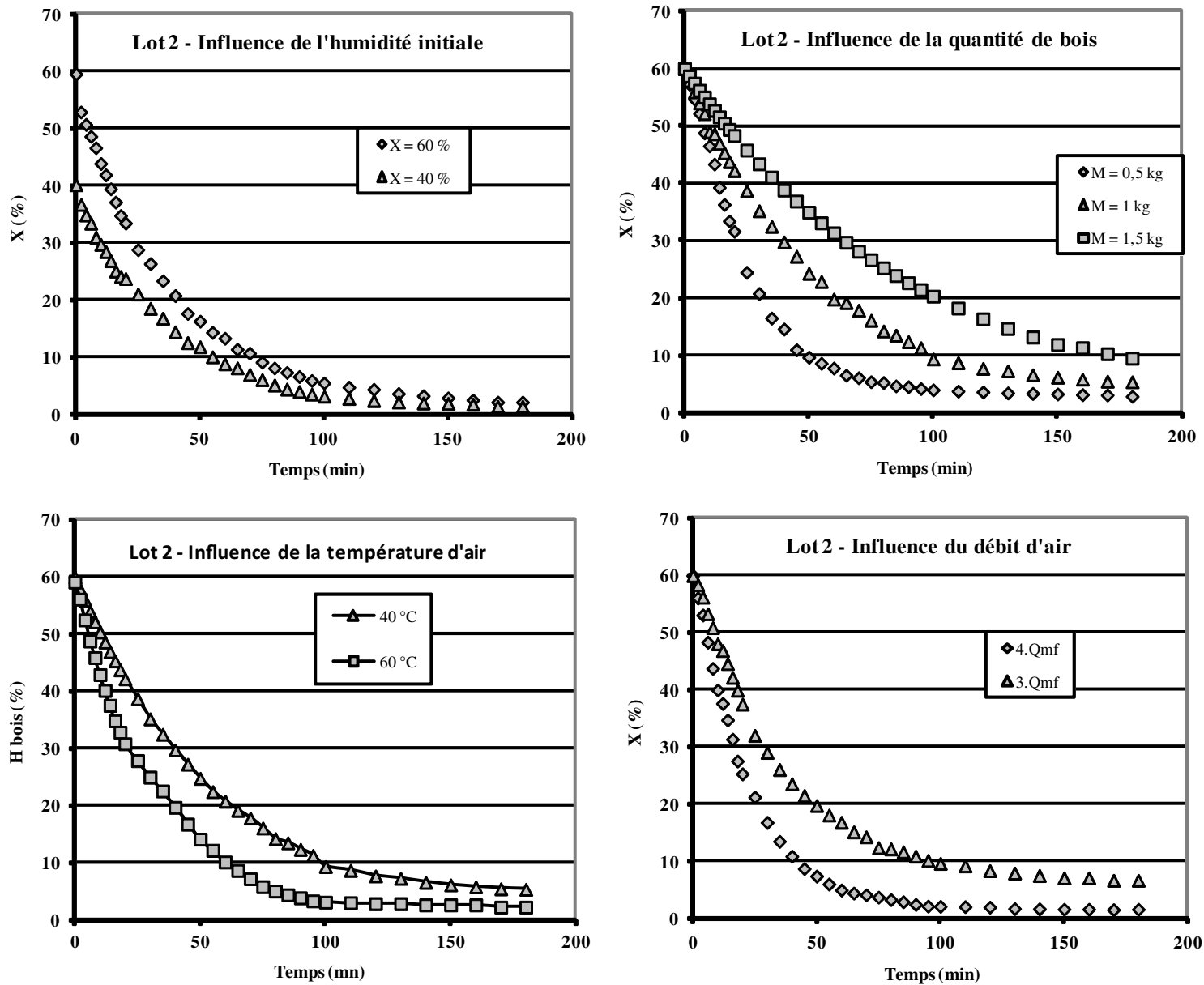

Fig. 6. Lot No. 2 - Évolution de la teneur en eau du bois (X) en fonction de la température et du débit d'air, de l'humidité initiale et de la quantité de bois.

de 0,5 fois le volume de sable qui correspond à une proportion en masse de 15 à $25 \%$.

D'autre part, l'humidité des particules de bois est ramenée à une valeur de $10 \%$ dans des temps inférieurs à $1 \mathrm{~h}$. Ceci est un paramètre important car la maîtrise de l'humidité est un critère primordial pour optimiser la combustion du bois (le bois vert broyé impose un séchage de 4 à 6 mois avant de passer en chaudière).

Si le temps nécessaire pour amener l'humidité du bois à une valeur de $10 \%$ caractérise la qualité du traitement opéré, il apparaît qu'elle est largement influencée par des paramètres liés au fonctionnement du réacteur (température et débit d'air) mais également à la charge (humidité initiale et quantité de bois) (Fig. 6).

Une température d'air supérieure à $60{ }^{\circ} \mathrm{C}$ avec un débit de 4 fois le débit minimal de fluidisation permet de réduire de $50 \%$ le temps de traitement. Une charge de bois faible (15 à $20 \%$ ), associée à un préséchage permet également d'améliorer la durée du séchage.

\section{Références}

[1] J.H.B.J. Hoebink, K. Rietema, Drying granular solids in fluidized bed, Chem. Eng. Sci. 35 (1980) 2135-3140
[2] H. Groenewald, E. Tsotas, Proc. 11th International Drying Symposium, Drying 98, Predicting apparent Sherwood-numbers for fluidized beds, ThessalonikiHalkidiki, Greece, 1998

[3] A.N. Chandra, S. Subba, Y.B.G. Varma, H. Aiche, J. Fluidized bed drying of solids 36 (1990) 29-38

[4] D.G. Dimattia, P.R. Amyotte, F. Hamdullahpur, Amer. Soc. Agricultural Eng., Fluidized bed drying of large particles 39 (1996) 1745-1750

[5] G. Rios, H. Gibert, Heat transfer between gaz fluidized bed and big bodies: analysis and explanation of big body mobility effects, 4th Int. Conference on Fluidization, Japan, 1983, pp. 363-369

[6] M. Mourad, M. Hemati, C. Laguerie, Séchage de maïs en lit fluidisé à flottation : détermination des vitesses caractéristiques de fluidisation de mélange de maïs et de sable, Powder Techn. 80 (1994) 45-54

[7] R. Bilbao, J.L. Lezaun, J.C. Abanades, Fluidization velocity of sand/straw binary mixtures, Powder Techn. 52 (1988) 1-6

[8] V. Thonglimp, Contribution à l'étude hydrodynamique des couches fluidisées par un gaz, vitesse minimale de fluidisation et expansion, Thèse de docteur Ingénieur de l'INP Toulouse, 1981 
[9] C.Y. Wen, Y.H. Yu, A generalized method for predicting the minimum fluidization velocity, American Institute of Chemical Engineers, 1966, pp. 610-612

[10] V.D. Goroshko et al., Approximate hydraulic relationships for suspends beds and hindered fall, Neft. I. Gas (1958) $125-131$

[11] B.C. Pillai, M. Raja Rao, Pressure drop and fluidization velocities in air-fluidized beds, Indian J. Technol. 9 (1971) $77-86$

[12] J. Baeyens, D. Geldart, La fluidisation et ses applications Cepadues Editions, Particles mixing in a gas fluidized bed, Toulouse, 1973 , pp. 182-195

[13] J.F. Richardson, M.A.S. Jeronimo, Velocity-voidage relations for sedimentation and fluidisation, Chem. Eng. Sci. 34 (1954) 1419-1422
[14] J. Bena, Chemcky Prumysl 10 (1960) 285

[15] M. Leva, T. Shirai, C.Y. Wen, La prévision du début de fluidisation dans les lits solides granulaires, Génie Chimique 75 (1956) 33-42

[16] T.H. Hsiung, G. Thodos, Expansion characteristcs of gasfluidized beds, Can. J. Chem. Eng. 55 (1977) 221-224

[17] E.W. Lewis, W. Bowermann, Chem. Eng. Progr. 48 (1952) 603

[18] S.P. Babu et al., Fluidization correlations for coal gasification material - minimum fluidisation velocity and fluidized bed expansion ratio 74 (1978) 176-186

[19] S. Strijbos, Motion and distribution of larges particles suspended in a fluidised bed, Powder Techno. 6 (1972) $337-342$ 